\title{
Geld oder Leben?
}

\author{
Money or Life?
}

\author{
Autor \\ M. Wildner \\ Institut \\ Bayerisches Landesamt für Gesundheit und Lebensmittelsicherheit, Oberschleissheim
}

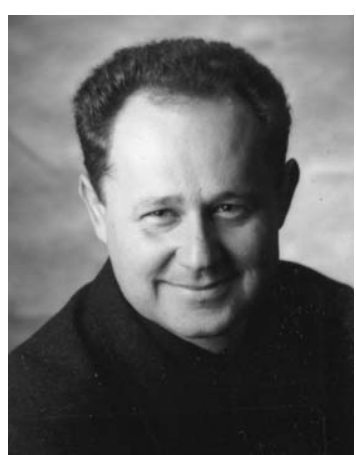

PD Dr. Manfred Wildner
„Geld oder Leben!“ - mit dieser unfreundlichen Alternative sehen sich die Opfer von Raubüberfallen konfrontiert, Variationen der Wortwahl außer Acht gelassen. Und damit wird von ihnen höchst unbequem die rasche Aufstellung einer Wertehierarchie verlangt. Den meisten ist das Leben lieber und die so Angesprochenen sind daher bereit, auf das Geld, das sie in der Tasche tragen, zu verzichten. Letztlich wird also der Grundwert „Recht auf Leben“ geschont, während der Grundwert „Recht auf Eigentum“ leidet. Der Angreifer wiederum mag sich in seiner freien Persönlichkeitsentfaltung bestätigt sehen, zumindest solange, bis er polizeilich festgesetzt und im Einklang mit dem Grundgesetz genau in diesem Grundrecht auf freie Persönlichkeitsentfaltung massiv beschnitten wird.

Was ist der Bezug zum Gesundheitswesen? Diese Frage kann hier nur angedacht werden. Derartige Wertekonflikte zeigen sich z.B. in den typischen Diskussionen um die Nichtraucherschutzgesetzgebungen der Länder. Die vorgetragenen Argumente sind wertebasiert: Auf Seiten der Nichtraucher und auch der Berufstätigen z.B. in der Gastronomie (Raucher und Nichtraucher) wird das Recht auf körperliche Unversehrtheit und Leben angeführt („Leben“), auf Seiten der Raucher das Recht auf freie Persönlichkeitsentfaltung (ebenfalls „Leben“) und auf Seiten der Gastwirte das Recht auf freie Berufsausübung (eine Mischung aus „Geld“ und „Leben“). Als viertes Argument werden gelegentlich die staatlichen Steuereinnahmen angeführt - der Raucher als „guter Staatsbürger“ im Sinne vermehrter Steuerabgaben und zeitlich verkürzter Rentenansprüche („Geld“). Die Gerichte setzen sich zunehmend mit derartigen Fragen auseinander.

In einem solchen Wertekonflikt müssen Abwägungen getroffen werden. Vor den Argumenten kommen bekanntlich die Fakten. Es steht zu befürchten, dass nicht jedes Gutachten ausreichend auf die umfassende Faktenlage einschließlich Ar- beits- und Jugendschutz eingeht. Welches sind wesentliche Fakten zur Nichtraucherschutzgesetzgebung? Bekannt ist, dass mindestens einer von drei Rauchern an den Folgen seines Tabakkonsums stirbt - bei Frauen womöglich zwei von drei $[1,2]$. Ebenfalls bekannt ist, dass dieses Sterberisiko in stärkerem Maß von Herz-KreislaufKrankheiten bestimmt wird als von Krebserkrankungen [3]. Jedoch erst in den letzten Jahren und im Zusammenhang mit der Einführung von Nichtraucherschutzgesetzgebungen insbesondere in der Gastronomie wurde deutlich, dass das Passivrauchen eine erheblich stärkere Gefährdung für die dem Tabakrauch Ausgesetzten bedeutet als bisher angenommen. Dies spiegelt sich nicht nur in dem überraschend hohen Rückgang der Herzinfarktraten in mehreren Ländern nach in Kraft Treten von Nichtraucherschutzgesetzen wieder, sondern auch in Metaanalysen aus dem arbeitsmedizinischen Bereich [4-10].

In Italien ist nach ersten Berichten das Herzinfarktrisiko um 10\%, in Frankreich um 15\% gesunken, nachdem ein Rauchverbot eingeführt wurde. Dabei ist keine wesentliche Reduzierung anderer Risikofaktoren als Erklärung bekannt. Aus den USA wurden Reduktionen um 27\% bzw. 40\% berichtet, diese sind jedoch möglicherweise eine methodenbedingte Überschätzung [11,12]. Überträgt man dies auf Deutschland, würden jährlich ca. 10000-15000 Herzinfarkt-Todesfälle durch die Nichtraucherschutzgesetzgebung verhindert werden. Hinzu kommen verhinderte Todesfälle bei Schlaganfall und Krebs.

Es wird davon ausgegangen, dass das erhöhte Sterberisiko bei Passivrauchexponierten fast so hoch ist wie bei den Rauchern (etwa 80-90\% des erhöhten kardiovaskulären Sterberisikos). Weltgesundheitsorganisation (WHO) und Europäische Union (EU) rufen angesichts solcher Fakten zu einem entschiedenen Vorgehen bei der Tabakkontrolle auf $[3,13]$. Auch die einschlägigen deutschen Fachgesellschaften haben einen Appell an 
die politischen Verantwortungsträger gerichtet. Sie fordern, die Nichtraucherschutzgesetzgebung in stringenter Form dringend beizubehalten [14]. Indirekte Evidenz zum Erfolg der Tabakkontrollmaßnahmen in Deutschland liegt vor: z. B. ist der Verbrauch an versteuerten Zigaretten in Deutschland seit Jahren deutlich rückläufig.

Vorbildlich ist das Vorgehen in Schottland. Dort werden die Auswirkungen der Nichtraucherschutzgesetzgebung zeitnah mit verschiedenen Evaluationsansätzen auf Bevölkerungsebene begleitet [15]. Ein solcher Ansatz „evidenzbasierter Gesundheitspolitik“ mit entsprechenden Forschungsaufträgen an einschlägige wissenschaftliche Institute ist zu begrüßen - wünschenswert wäre dabei auch die Einbeziehung ethischer Gesichtspunkte der Güterabwägung („Geld oder Leben“).

Ebenfalls eine Güterabwägung stellt die für dieses Heft in drei Beiträgen aufgegriffene Diskussion zur These „,Mehr Markt macht nicht gesund“ dar - sicher auch für Sie spannend zu lesen!

\section{Literatur}

1 Doll R, Peto R, Boreham J, Sutherland I. Mortality in relation to smoking: 50 years observations on male British doctors. Br Med J 2004; 328 : 1519 doi:10.1136/bmj.38142.554479.AE [published 22 June 2004]

2 Kenfield SA, Stampfer MJ, Rosner BA, Colditz GA. Smoking and smoking cessation in relation to mortality in women. JAMA 2008; 299 (17): 2037-2047

3 WHO. WHO report on the global tobacco epidemic, 2008. The MPOWER package. Geneva, WHO 2008, (s.a. http://www.who.int/tobacco/ mpower/mpower_report_full_2008.pdf])

4 Juster HR, Loomis BR, Hinman TM, Farrelly MC, Hyland A, Bauer UE, Birkhead GS. Declines in hospital admissions for acute myocardial infarction in New York State after implementation of a comprehensive smoking ban. Am J Public Health 2007; 97 (11): 2035-2039
5 Glantz FK, Glantz SA. Protecting Europeans from secondhand smoke. Eur Heart J 2006; 27: 382-383

6 Pechacek TF, Babb S. How acute and reversible are the cardiovascular risks of secondhand smoke? Br Med J 2004; 328: 980-983

7 Haw S, Gruer L. Changes in exposure of adult non-smokers to secondhand smoke after implementation of smoke-free legislation in Scotland: national cross sectional survey. Br Med J 2007; 335: 549-552

8 Tong EK, Glantz SA. Tobacco industry efforts undermining evidence linking secondhand smoke with cardiovascular disease. Circulation 2007: 116: 1845-1854

9 Barnoya J, Glantz SA. Cardiovascular effects of secondhand smoke: nearly as large as smoking. Circulation 2005; 111: 2684-2698

10 Bartecchi C, Alsever RN, Nevin-Woods C, Thomas WM, Estacio RO, Bartelson BB, Krantz MJ. Reduction in the incidence of acute myocardial infarction associated with a citywide smoking ordinance. Circulation 2006; 114: 1490-1496

11 Cesaroni G, Forastiere F, Agabiti N, Valente P, Zuccaro P, Perucci CA. Effect of the Italian smoking ban on population rates of acute coronary events. Circulation 2008; 117 (9): 1183-1188

12 Khuder SA, Milz S, Jordan T, Price J, Silvestri K, Butler P. The impact of a smoking ban on hospital admissions for coronary heart disease. Prev Med 2007; 45: 3-8

13 http://ec.europa.eu/health/ph_determinants/life_style/Tobacco/ tobacco_en.htm , Zugriff am 16.06.2008

14 Robra BP, Schwartz FW, Walter K. Erfolgreiche Maßnahme zum Schutz der Gesundheit - BVÖGD, DGPH und DGSMP fordern weiterhin konsequenten Schutz vor den Gesundheitsgefahren durch Passivrauchen in öffentlichen Räumen statt Aufweichung geltender Rauchverbote in Deutschland. Gesundheitswesen 2008; 3: 183

15 Haw SJ, Gruer L, Amos A, Currie C, Fischbacher C, Fong GT, Hastings G Malam S, Pell J, Scott C, Semple S. Legislation on smoking in enclosed public places in Scotland: how will we evaluate the impact? J Public Health 2006; 28 (1): 24-30 